\title{
Validity and Reliability of the Hausa Version of Multidimensional Scale of Perceived Social Support Index
}

\author{
Ashiru Hamza Mohammad ${ }^{1, *}$; Nabilla Al Sadat ${ }^{1}$; Loh Siew Yim ${ }^{2}$; Karuthan Chinna ${ }^{1}$ \\ ${ }^{1}$ Department of Social and Preventive Medicine, University of Malaya, Kuala Lumpur, Malaysia \\ ${ }^{2}$ Department of Rehabilitation Medicine, University of Malaya, Kuala Lumpur, Malaysia \\ *Corresponding Author: Ashiru Hamza Mohammad, Department of Social and Preventive Medicine, University of Malaya, P. O. Box: 50603, Kuala Lumpur, Malaysia. Tel: +60- \\ 146396673, E-mail: physioash@yahoo.com
}

Received: March 8, 2014; Revised: May 24, 2014; Accepted: August 2, 2014

\begin{abstract}
Background: Social support has been identified as one of the key factors for enhancing the quality of life after stroke. However, a scientific tool that is valid for evaluating social support among stroke survivors in Nigeria has not been developed so far.

Objectives:The objective of this study wasto examine thepsychometric properties of theHausa translated versions of theMultidimensional Scale of Perceived Social Support(MSPSS) and to validate it for future use in clinical research in Nigeria.

Patients and Methods: This cross-sectional study was conducted on a sample of 140 adult stroke survivors attending rehabilitation services at stroke referral centers in Kano, Nigeria. The psychometric analysis of the Hausa-MSPSS involved face, content, criterion and construct validity tests, as well as internal and test-retest reliability. Confirmatory factor analysis was performed to assess validity of the three-factor (Family, Friends and Significant others) model for the Hausa-MSPSS.

Results: Based on expert panel, clinicians' review and patients' feedback, the 12 item Hausa-MSPSS had sufficient face, content and criterion validity. In reliability analysis, the Cronbach's alpha was 0.781 . In test-retest reliability analysis, the minimum kappa value was 0.673. In Confirmatory factor analysis, the three-factor model was not acceptable. An alternative two-factor model with Family and Friends, as the two domain was found to be acceptable (Chi-square/df $<3$, TLI, CFI $>0.9$, RMSEA $<0.08$ ). The average variances extracted for the two constructs were 0.5 and 0.7 , respectively. The R-squared value between the two constructs was 0.397 . Cross validity tested using 1000 bootstrap resamples gave satisfactory results $(\mathrm{P}=0.079)$.

Conclusions: The 11 item Hausa-MSPSS index is valid for the assessment of perceived social support among stroke survivors in Nigeria.
\end{abstract}

Keywords:Stroke; Social Support; Reliability and Validity

\section{Background}

The impact of stroke can be devastating, leaving a person with residual impairment of physical, psychological, and social functions $(1,2)$. During the sub-acute phase of stroke, when the potential for further functional gain is limited, the psychosocial consequences of stroke become increasingly important determinants of health-related quality of life (HRQOL) $(3,4)$. It has been shown that about one-third of individuals with stroke may develop post-stroke depression and perceived social support may be preventive for either onset or duration of post-stroke depression (3).

For stroke survivors, social support is believed to affect the quality of patient care and disease outcome, as well as patients' physical and psychosocial well-being $(4,5)$. Studies have consistently demonstrated a link between low levels of social support and poor mental and physical health outcomes and have subsequently fuelled the development and evaluation of interventions designed to improve social support for those deprived of this resource $(3,4,6,7)$. Apart from disability, psychosocial factors, such as emotional responses and social support, are important determinants of HRQOL in stroke survivors (8). In the broader sense, social support cuts across any process through which social relationships might promote health and well-being (9) and moderates the impact of limited functional status on well-being $(5,10)$. The improvement of accessible social support could be an essential means for mitigating psychiatric distress and averting post-stroke depression (3).

While the role of social support, as a coping resource, has generated increased interest in recent years (7), there is also a recent growing impetus in the use of the Multidimensional Scale of Perceived Social Support (MSPSS) to evaluate perceived social support across cultures (11). Although MSPSS was initially designed to assess perceptions of social support adequacy among adolescents from three different sources (family, friends, and significant others), a growing body of evidence on the utility of the MSPSS revealed that it is a useful measure for various populations, including cardiovascular disease patients and the elderly $(12,13)$. However, no tools or translated scales have been developed for the assessment of social 
support among stroke survivors in Nigeria. Since MSPSS was developed in English (Appendix 1), it was essential to carry out a translation and trans-cultural adaptation of the questionnaire where it was intended to be used.

\section{Objectives}

The aim of this study was to examine the psychometric properties of the MSPSS among Nigerians by using the Hausa translated versions of the MSPSS and to validate it for future use in clinical research and practice in Nigeria.

\section{Patients and Methods}

This cross-sectional study was conducted in the departments of Medicine and Physiotherapy of Aminu Kano Teaching Hospital (AKTH), Murtala Mohammad Specialist Hospital (MMSH) and Mohammad Abdullahi Wase Specialist Hospital (MAWSH) in Kano state, Nigeria. The AKTH is a tertiary care hospital with counting approximately 1000 beds. It provides high level medical care and receives patients from within Kano and neighboring states. The MMSH (700 bed capacity) and MAWSH (200 bed capacity) are specialty and referral hospitals located in Kano metropolis, which receive patients from within Kano. These three hospitals are the only hospitals in Kano that manage referred stroke patients and provide rehabilitation medical care. This was a universal sampling of consecutive patients with the diagnosis of stroke admitted or referred into the hospitals from 1st December 2010 to 30th January 2012. Inclusion criteria were: first-time stroke, age $\leq 18$ years and were receiving rehabilitation services after their overall condition was stabilized. Patients with a history of recurrent stroke attacks and persistent deficits, with underlying psychotic and mental disorders, those who were handicapped before the stroke event, with impaired ability to understand the questionnaire, such as those with severe aphasia, and the patients with comorbidities (e.g. heart failure, peritoneal or hemodialysis) that significantly affect the QOL and limit life expectancy, were excluded. Consecutive stroke patients who satisfied the inclusion criteria were recruited from the three hospitals until the desired sample size of 140 was obtained. This is a validation study with 12 items. As a rule of thumb, with 10 cases per construct (14), the total required sample size is 120 . In this study, a sample size of 140 was used. Written informed consent was also obtained for each patient prior to participation. Participants responding to the questionnaire could not go further in the questionnaire without selecting an option in every item, so there were no missing values.

The procedure involved in the development of the Hausa version of MSPSS included the process of linguistic validations, which was published elsewhere (15) and the subsequent psychometric validations of the Hausa-MSPSS. These cognitive processes were to ensure that the Hausa-MSPSS was measuring the same concept as the original scale, thereby enabling comparisons between different cultures.
The translated Hausa-MSPSS questionnaire (15) was administered among 35 stroke patients. These patients returned to the clinic again to complete the Hausa MSPSS one week after the first evaluation. The data collected were used in reliability and test-retest reliability analyses. Finally, the psychometric properties of the translated Hausa-MSPSS were assessed using data collected from 140 stroke survivors. Using AMOS 18 (Amos Development Corp., Crawford, FL, USA), a series of confirmatory factor analyses (CFAs) were performed to examine the nature of and relations among latent constructs. The CFA categorically tests a prio$r i$ hypotheses about relations between observed variables and latent variables or factors $(16,17)$. Besides testing the questionnaire, the feasibility of administering the questionnaire under field conditions was also noted.

\subsection{Description of the Survey Instrument}

The MSPSS (Appendix 1) is a well validated and psychometrically rigorous 12 items concise instrument used for measuring the hierarchical structure of perceived social support from family (items 3, 4, 8 and 11), friends (items 6, 7, 9, and 12), and significant others (items 1, 2, 5, and 10) (11, 13). Although it was developed for adolescents, it is also suitable for other groups. It has a number of properties which suggest that it may be a valuable tool for research use which involve a variety of populations, including cardiovascular disease patients and the elderly (11, 13, 18). Studies indicate that MSPSS has a good internal reliability, test-retest reliability, and strong factorial validity. In addition, good construct validity of the significant other, family, and friend subscales was also demonstrated $(7,12,19)$. The perceived social support is graded on a 7-point Likert-type scale (from very strongly disagree to very strongly agree) as follows: respondents are required to circle: 1- very strongly disagree, 2 - strongly disagree, 3- mildly disagree, 4- neutral, 5- mildly agree, 6- strongly agree or 7- very strongly agree.

\subsection{Ethical Considerations}

The study protocol was approved by the Medical Ethics Committee of the University of Malaya, Malaysia (Ethics Committee/IRB Ref. number 830.7), and also from the Ministry of Health of Kano State, Nigeria (HMB/ GEN/488/11) and Aminu Kano Teaching Hospital of Nigeria (AKTH/MAC/SUB/12A/P3/IV/801).

\subsection{Data Analysis}

The psychometric analysis of the Hausa-MSPSS instrument involved the assessment of internal consistency, test-retest reliability, and content, face, criterion, as well as construct, validity. Double data entry was carried out and cross-checked to assure the consistency and quality of the data. Analysis was carried out using the statistical program SPSS for Windows version 20.0 (SPSS Inc., Chicago, ILL, USA). The face and content validity were tested through a pilot test involving stroke patients during the linguistic 
validation process by the experts in QOL measures, public health and stroke rehabilitation. The criterion validity of the MSPSS was assessed regarding its ability to measure what was it intended to measure (14). In this study, MSPSS intended to measure the perceived social support in stroke survivors. Finally, a series of confirmatory factor analyses were performed using the AMOS 18 software (Amos Development Corp., Crawford, FL, USA) to determine if the number of factors and the loadings measured (indicator) variables on them conform to what is expected on the basis of the pre-established theory (20). In this model, there were no mediator variables. The purpose of this method is to validate the constructs, not to test relationships.

\section{Results}

The study was conducted on 140 stroke survivors. The sample was predominantly Hausa ( $n=136,97.1 \%)$, three, Yoruba (2.1\%) and one, other linguistic group (0.7\%). There were slightly more males $(n=75,53.6 \%)$ than females ( $n$ $=65,46.4 \%$ ) and their ages ranged between $40-80$ years, with a mean age of $58.76 \pm 13.24$ years. Sixty-six (47.1\%) patients had left hemisphere lesions, whereas 74 (52.9\%) had right hemisphere involvement. Cerebral ischemic stroke was detected in 57 (40.7\%) patients, cerebral hemorrhage in 18 (12.9\%) and indeterminate in 65 (46.4\%) patients. Most of the participants were residents in urban areas $(n=102,72.9 \%)$.

The content and face validity testing of the Hausa- MSPSS index was performed by a group of subject experts. According to these experts, the Hausa-MSPSS index had achieved conceptual, semantic and operational equivalence with the original index. During translation, and where required, expressions used in the items were subjected to more culturally acceptable linguistic equivalents, similar in concept and meaning to the original items. A pretesting and pilot study with 30 stroke survivors additionally attested the face and content validation of the Hausa-MSPSS index. The feasibility of administering the instrument under field condition was also verified. The instrument was found to be easily conceivable, simple, clear and appropri- ate for the assessment of social support among this group of stroke survivors. Moreover, the time taken to answer the instrument (5-10 minutes) was acceptable.

In reliability analysis, the Cronbach's alpha for the 12 items was 0.781. The Cronbach's alpha values for perceived social support from family, friends and significant others were 0.827 , friends 0.898 and 0.779 , respectively. In testretest reliability analysis, the minimum kappa value was 0.673 , as shown in Table 1 . In this model, there were no mediating variables. The purpose was to validate the constructs, and not to test relationships. For all items, the ceiling effects were 7. For three out of the 12 items, the floor effects were 2. For significant others, it was 1 . In CFA, testing the adequacy of a three-factor model, the correlation between family and significant others constructs was 0.91 , as can be seen in Figure 1, indicating the phenomenon of multicollinearity (14) between these two constructs.

In these situations, either one of the constructs must be dropped, or the items in the two constructs must be combined into one (21). In this study, the second option was considered. Items from the two constructs (family and significant others) were combined into one, and the name of the construct was maintained as family. Item four (I get the emotional help and support I need from my family) lacked discriminant validity and hence, it was dropped. The two factor model (Figure 2), with just 11 items, was found to be acceptable CChi-square/df $<3$, TLI and CFI > 0.9, RMSEA < 0.08). The Critical value for multivariate kurtosis value was greater than five. Hence, multivariate normality assumption was not met. Therefore, the model was validated using 1000 bootstrap resamples. The test gave a satisfactory result $(P=0.079)$. The minimum factor loading in the two-factor model was 0.667. The average variance extracted (AVE) for the family and friends constructs were 0.5 and 0.7 , respectively. The R-squared value between the two constructs was 0.397 . Since the R-squared value is less than the AVEs, there is sufficient discriminant validity between the two constructs. The factor loadings for the two constructs are provided in Table 2 the final path diagram is shown in Figure 2.

\begin{tabular}{|c|c|c|c|}
\hline Item & Description & $\mathbf{r s}^{\mathrm{a}}$ & Kappa \\
\hline SS 1 & There is a special person who is around when I am in need & 0.883 & 0.673 \\
\hline SS 2 & There is a special person with whom I can share my joys and sorrows & 0.949 & 0.806 \\
\hline SS 3 & My family really tries to help me & 0.924 & 0.702 \\
\hline SS 4 & I get the emotional help and support I need from my family & 0.955 & 0.814 \\
\hline SS 5 & I have a special person who is a real source of comfort to me & 0.970 & 0.854 \\
\hline SS 6 & My friends really try to help me & 0.971 & 0.813 \\
\hline SS 7 & I can count on my friends when things go wrong & 0.983 & 0.888 \\
\hline SS 8 & I can talk about my problems with my family & 1.000 & 1.000 \\
\hline SS 9 & I have friends with whom I can share my joys and sorrows & 0.978 & 0.854 \\
\hline SS 10 & There is a special person in my life that cares about my feelings & 0.970 & 0.895 \\
\hline SS 11 & My family is willing to help me make decisions & 1.000 & 1.000 \\
\hline SS 12 & I can talk about my problems with my friends & 0.922 & 0.711 \\
\hline
\end{tabular}

a rs: Spearman's correlation. 
Mohammad AH et al.

\begin{tabular}{|c|c|c|c|c|c|c|}
\hline Items & & Domains & Unstd. Estimate & S.E. & C.R. & Std Estimate \\
\hline SS11 & $<-$ & Family & 1.000 & & & 0.787 \\
\hline SS8 & $<-$ & Family & 0.922 & 0.097 & 9.514 & 0.667 \\
\hline SS3 & $<-$ & Family & 0.913 & 0.089 & 10.251 & 0.711 \\
\hline SS1 & $<-$ & Family & 0.907 & 0.105 & 8.660 & 0.614 \\
\hline SS2 & $<-$ & Family & 1.011 & 0.094 & 10.798 & 0.744 \\
\hline SS5 & $<-$ & Family & 0.967 & 0.093 & 10.345 & 0.717 \\
\hline SS10 & $<-$ & Family & 1.044 & 0.104 & 10.054 & 0.699 \\
\hline SS12 & $<-$ & Friends & 1.000 & & & 0.878 \\
\hline SS9 & $<-$ & Friends & 0.952 & 0.065 & 14.679 & 0.825 \\
\hline SS7 & $<-$ & Friends & 0.957 & 0.065 & 14.650 & 0.824 \\
\hline SS6 & $<-$ & Friends & 0.963 & 0.066 & 14.517 & 0.820 \\
\hline
\end{tabular}

a P value $<0.001$.

b Abbreviations: CR, censored regression; SE, standard error; Std., standardized; Unstd., Unstandardized.
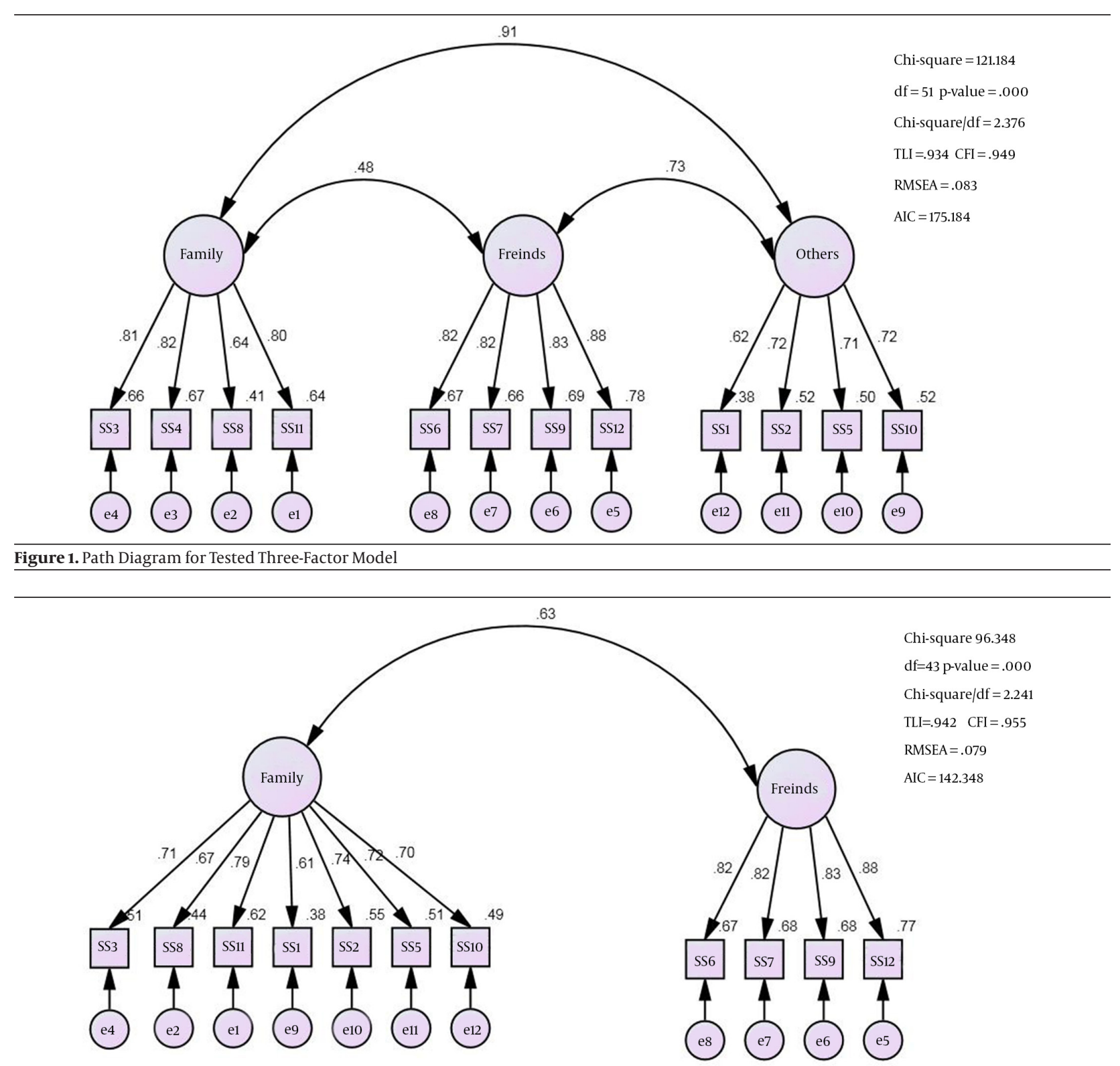


\section{Discussion}

The development of the Hausa version of the MSPSS was conducted according to the established protocols by the Mapi Research Institute's methodology 2004 (22), which is also similar to the guidelines provided by Beaton et al. (2000) (23) for the cross-cultural adaptation of selfreported measures. The protocol consists of translation, synthesis, back translation, expert committee review and pilot testing. The MSPSS validation was conducted in close collaboration between the translation committee comprising the multiprofessional experts from University of Malaya, Bayero University Kano, Nigeria and professional translators from the Freedom Radio Nigeria muryar jama'a 99.5FM (an independent radio) and the copyright owner/ developers of the questionnaire. It was found that the final Hausa version of the MSPSS had equivalent subject content and meaning and closely similar wordings to the original English version. It was also found to be easily conceivable, simple, clear and appropriate for the assessment of social support among the group of stroke survivors in Nigeria. Its psychometric properties, in terms of content, face, construct and criterion validity, as well as internal, test-retest reliability, had been tested and verified.

This study is the first of its kind in Nigeria that involved translation, testing and evaluation of the psychometric properties of the Hausa version of the MSPSS. According to the Clarke and Watson (24), the number of items in an instrument determines the sample size to be used in the psychometric testing. For an instrument with 20 items or less, a sample size between 100 and 200 subjects is deemed to be adequate (25). Hence, the sample size of 140 used in this study is considered to be adequate for testing the psychometric properties of the Hausa-MSPSS. Furthermore, the subjects were chosen randomly.

In the reliability analysis, the Cronbach's alpha for the 12 items was 0.781 . All items correlated well with the to- tal score and the scale, overall. In test-retest reliability analysis, the minimum kappa value was 0.67 . This also indicates that the index had been proven to be reliable in yielding rational scores and was highly reliable to be used in the Nigerian setting. In CFA, a problem of multicollinearity between the items of the two constructs (family and significant others) was detected. In the region where the study was conducted, significant others and family are culturally regarded as family, and therefore the items of the two constructs were combined into one and the name of the resulting construct was maintained as family. After dropping one item, a two factor model with just 11 items was found to be acceptable (Chi-square/df $<3$, TLI and CFI > 0.9, RMSEA < 0.08).

The strength of our study relied on the fact that the study involved a rigorous forward-backward translation of the tool. An extensive AMOS modeling was run to confirm the factor structure, and, in the end, we obtained a validated instrument. The study had several limitations. Firstly, although we have an adequate sample size, a larger study population would have strengthen the results. Secondly, the measure was not tested against any measure of perceived social support, an aspect that should be take into consideration by future studies.

In conclusion, the present study revealed that the 11 item Hausa-MSPSS (Appendix 2) index is valid and can reliably be used to assess perceived social support among stroke survivors in Nigeria. It is hoped that in the future, researchers investigating perceived social support from family, friends and significant others in stroke survivors will use this measure in their studies. With this valid instrument, further improvement in mitigating psychiatric distress and averting post-stroke depression would be possible and planned.

\section{Appendix}

Appendix 1 English Version of the Multidimensional Scale of Perceived Social Support Multidimensional Scale of Perceived Social Support $^{\mathrm{a}}$

\begin{tabular}{|c|c|c|c|c|c|c|c|c|}
\hline & Perceived Support From Family, Friends and Significant Others & & & & le & & & \\
\hline 1 & There is a special person who is around when I am in need & 7 & 6 & 5 & 4 & 3 & 2 & 1 \\
\hline 2 & There is a special person with whom I can share my joys and sorrows & 7 & 6 & 5 & 4 & 3 & 2 & 1 \\
\hline 3 & My family really tries to help me & 7 & 6 & 5 & 4 & 3 & 2 & 1 \\
\hline 4 & I get the emotional help and support I need from my family & 7 & 6 & 5 & 4 & 3 & 2 & 1 \\
\hline 5 & I have a special person who is a real source of comfort to me & 7 & 6 & 5 & 4 & 3 & 2 & 1 \\
\hline 6 & My friends really try to help me & 7 & 6 & 5 & 4 & 3 & 2 & 1 \\
\hline 7 & I can count on my friends when things go wrong & 7 & 6 & 5 & 4 & 3 & 2 & 1 \\
\hline 8 & I can talk about my problems with my family & 7 & 6 & 5 & 4 & 3 & 2 & 1 \\
\hline 9 & I have friends with whom I can share my joys and sorrows & 7 & 6 & 5 & 4 & 3 & 2 & 1 \\
\hline 10 & There is a special person in my life that cares about my feelings & 7 & 6 & 5 & 4 & 3 & 2 & 1 \\
\hline 11 & My family is willing to help me make decisions & 7 & 6 & 5 & 4 & 3 & 2 & 1 \\
\hline 12 & I can talk about my problems with my friends & 7 & 6 & 5 & 4 & 3 & 2 & 1 \\
\hline
\end{tabular}

\footnotetext{
${ }^{\mathrm{a}}$ Instructions: We are interested in how you feel about the following statements. Read each statement carefully. Indicate how you feel about each statement. Circle the " 1 " if you Very Strongly Disagree Circle the " 2 " if you Strongly Disagree Circle the " 3 " if you Mildly Disagree Circle the " 4 " if you are Neutral Circle the "5" if you Mildly Agree Circle the "6" if you Strongly Agree Circle the "7" if you Very Strongly Agree.
} 
Mohammad AH et al.

\begin{tabular}{|c|c|c|c|c|c|c|c|c|}
\hline \multirow[b]{2}{*}{$\mathbf{1}$} & \multirow{2}{*}{$\begin{array}{c}\text { Perceived Support From Family, Friends and Significant Others } \\
\text { There is a special person who is around when I am in need }\end{array}$} & \multicolumn{7}{|c|}{ Scale } \\
\hline & & 7 & 6 & 5 & 4 & 3 & 2 & 1 \\
\hline 2 & There is a special person with whom I can share my joys and sorrows & 7 & 6 & 5 & 4 & 3 & 2 & 1 \\
\hline 3 & My family really tries to help me & 7 & 6 & 5 & 4 & 3 & 2 & 1 \\
\hline 4 & I have a special person who is a real source of comfort to me & 7 & 6 & 5 & 4 & 3 & 2 & 1 \\
\hline 5 & My friends really try to help me & 7 & 6 & 5 & 4 & 3 & 2 & 1 \\
\hline 6 & I can count on my friends when things go wrong & 7 & 6 & 5 & 4 & 3 & 2 & 1 \\
\hline 7 & I can talk about my problems with my family & 7 & 6 & 5 & 4 & 3 & 2 & 1 \\
\hline 8 & I have friends with whom I can share my joys and sorrows & 7 & 6 & 5 & 4 & 3 & 2 & 1 \\
\hline 9 & There is a special person in my life that cares about my feelings & 7 & 6 & 5 & 4 & 3 & 2 & 1 \\
\hline 10 & My family is willing to help me make decisions & 7 & 6 & 5 & 4 & 3 & 2 & 1 \\
\hline 11 & I can talk about my problems with my friends & 7 & 6 & 5 & 4 & 3 & 2 & 1 \\
\hline
\end{tabular}

a Instructions: We are interested in how you feel about the following statements. Read each statement carefully. Indicate how you feel about each statement. Circle the "1" if you Very Strongly Disagree Circle the "2" if you Strongly Disagree Circle the "3" if you Mildly Disagree Circle the " 4 " if you are Neutral Circle the " 5 " if you Mildly Agree Circle the "6" if you Strongly Agree Circle the " 7 " if you Very Strongly Agree.

\section{Acknowledgements}

The authors would like to especially thank the participants and staff of Aminu Kano Teaching Hospital and Murtala Mohammad Specialist Hospital, and Muhammad Abdullahi Wase Specialist Hospital Kano, Nigeria for their active involvement in the study.

\section{Authors' Contributions}

Study concept and design: Ashiru Hamza Mohammad, Nabilla Al Sadat, Loh Siew Yim; analysis and interpretation of data: Karuthan Chinna, Ashiru Hamza Mohammad, Nabilla Al Sadat, Loh Siew Yim; drafting of manuscript: Ashiru Hamza Mohammad, Karuthan Chinna, Nabilla Al Sadat and Loh Siew Yim. Ashiru Hamza Mohammad developed the original idea and wrote the manuscript. Assoc. Prof. Nabilla Al Sadat and Dr Loh Siew Yim contributed to the development of the protocol and in preparing the manuscript and supervision. Assoc. Prof. Kauthan Chinna helped in the Study concept and design, acquisition of data, analysis and interpretation of data.

\section{Funding/Support}

This study is supported by the University of Malaya Postgraduate Research Grant (project number: PS034-2012A).

\section{References}

1. Lai SM, Perera S, Duncan PW, Bode R. Physical and social functioning after stroke: comparison of the Stroke Impact Scale and Short Form-36. Stroke. 2003;34(2):488-93.

2. Carod-Artal FJ, Trizotto DS, Coral LF, Moreira CM. Determinants of quality of life in Brazilian stroke survivors. J Neurol Sci. 2009;284(1-2):63-8

3. Salter K, Foley N, Teasell R. Social support interventions and mood status post stroke: a review. Int J Nurs Stud. 2010 47(5):616-25.
4. Lynch TR, Mendelson T, Robins CJ, Krishnan KR, George LK, Johnson CS, et al. Perceived social support among depressed elderly, middle-aged, and young-adult samples: cross-sectional and longitudinal analyses. J Affect Disord. 1999;55(2-3):159-70.

5. Tsouna-Hadjis E, Vemmos KN, Zakopoulos N, Stamatelopoulos S. First-stroke recovery process: the role of family social support. Arch Phys Med Rehabil. 2000;81(7):881-7.

6. Hogan BE, Linden W, Najarian B. Social support interventions: do they work? Clin Psychol Rev. 2002;22(3):383-442.

7. Bruwer B, Emsley R, Kidd M, Lochner C, Seedat S. Psychometric properties of the Multidimensional Scale of Perceived Social Support in youth. Compr Psychiatry. 2008;49(2):195-201.

8. Owolabi MO. Determinants of health-related quality of life in Nigerian stroke survivors. Trans $R$ Soc Trop Med Hyg. 2008;102(12):1219-25.

9. Colella TJ, King KM. Peer support. An under-recognized resource in cardiac recovery. Eur J Cardiovasc Nurs. 2004;3(3):211-7.

10. Clarke P, Marshall V, Black SE, Colantonio A. Well-being after stroke in Canadian seniors: findings from the Canadian Study of Health and Aging. Stroke. 2002;33(4):1016-21.

11. Cheng ST, Chan ACM. The multidimensional scale of perceived social support: dimensionality and age and gender differences in adolescents. Pers Indiv Differ. 2004;37(7):1359-69.

12. Tonsing K, Zimet GD, Tse S. Assessing social support among South Asians: the multidimensional scale of perceived social support Asian J Psychiatr. 2012;5(2):164-8.

13. Edwards LM. Measuring Perceived Social Support in Mexican American Youth: Psychometric Properties of the Multidimensional Scale of Perceived Social Support. Hispanic J Behav Sci. 2004;26(2):187-94

14. Hair JF, Black WC, Babin BJ, Anderson RE. Multivariate Data Analy sis. 7 edNew Jersey: Prentice Hall; 2010.

15. Hamza A, Al-Sadat Abdul Mohsein N, Yim LS. Measuring percieved social support in stroke survivors: linguistic validation of the Multidimensional Scale of Percieved Social Support (MSPSS) in Hausa (Nigerian) language. S Afr J Occup Ther. 2012;42(3):26-31.

16. Field A. Discovering Statistics Using SPSS. 3 ed: SAGE Publications, 2009.

17. Jackson DL, Gillaspy JA, Purc-Stephenson R. Reporting practices in confirmatory factor analysis: an overview and some recommendations. Psychol Methods. 2009;14(1):6-23.

18. Ekback M, Benzein E, Lindberg M, Arestedt K. The Swedish version of the multidimensional scale of perceived social support (MSPSS)--a psychometric evaluation study in women with hirsutism and nursing students. Health Qual Life Outcomes. 2013;11:168.

19. White JH, Alston MK, Marquez JL, Sweetapple AL, Pollack MR, 


\section{Mohammad AH et al.}

Attia J, et al. Community-dwelling stroke survivors: function is not the whole story with quality of life. Arch Phys Med Rehabil. 2007;88(9):1140-6.

20. Brown TA. Confirmatory Factor Analysis for Applied Research. In: Field AP editor. Discovering statistics using SPSS. 3 ed. New York: Sage publications; 2009.

21. Bove LL, Pervan SJ, Beatty SE, Shiu E. Service worker role in encouraging customer organizational citizenship behaviors. J Bus Res. 2009;62(7):698-705.

22. Acquadro C, Conway K, Girourdet C, Mear I. Linguistic validation manual for patient-reported outcomes (PRO) instruments.: Mapi Research Institute; 2004.

23. Beaton DE, Bombardier C, Guillemin F, Ferraz MB. Guidelines for the process of cross-cultural adaptation of self-report measures. Spine (Phila Pa 1976). 2000;25(24):3186-91.

24. Clark LA, Watson D. Constructing validity: Basic issues in objective scale development. Psychological assessment. 1995;7(3):309.

25. Yus of ZY, Jaafar N. A Malay version of the Child Oral Impacts on Daily Performances (Child-OIDP) index: assessing validity and reliability. Health Qual Life Outcomes. 2012;10:63. 